\title{
ANEURYSMAL BONE CYST OF THE CALCANEUM-A CASE SERIES
}

\author{
Orthopaedics \\ Dr.Dibakar ray \\ Prof,dept Of Orthopaedics, Iqcity Medical College,durgapur
}

\section{ABSTRACT}

Aneurysmal bone cyst $(\mathrm{ABC})$ is a benign bone tumor:

Background:It is mainly repoted in the long bones but only rare cases in calcaneum have been reported. As per reports only $1 \%$ of this type of tumour is found in the calcaneum

Material and Methods: I am going to report an unusual case series of ABC in calcaneum in 8 cases in last 7 years of which in male patients of young adults were predominant. All patients throughly investigated as per protocol set in study and treated accordingly with biopsy and through currattage and bone grafting uniformly.

Results:All patients was achieved proper diagnosis and good clinical results.

Conclusion:This article discusses regarding rare presentation of 8 cases of anurysmal bone cyst of calcaneum in the study period of 7 years with the etiology clinicapresentation, radiological features, histological features and preferred examinations with treatment done with results.

\section{KEYWORDS}

Anurysmal bone cyst, Calcaneum, 8 cases ,biopsy,currattage.

\section{Background:}

Anurysmal bone cyst is very uncommon pathological entity is calcaneum bone.It needs high end suspicion and radioclinico histological confirmation.

In this uncommon scenario 8 cases in last 7 years is received

\section{AIMS AND OBJECTIVES:}

Aim and objective is to publish very uncommon pathology of calcaneun that is anurysmal bone cyst in 8 nunbers in last 7 years of clinical practice

\section{INTRODUCTION}

The aneurysmal bone cyst $(\mathrm{ABC})$ is an expansile cystic lesion that most often affects individuals during their second decade of life and may occur in any bone in the body,but long bones are mostly involved ( 14) Although benign, the $\mathrm{ABC}$ can be locally aggressive and can cause extensive weakening of the bony structure and impinge on the surrounding soft tissues. Jaffe and Lichtenstein first described ABC in $1942,(! !)$. As defined by the World Health Organization, the ABC is a benign tumor like lesion described as "osteolytic lesion consisting of blood-filled spaces of variable size separated by connective tissue septa containing trabeculae or osteoid tissue and osteoclast giant cells, (1). ABCs both erode and cause 'expansion' of underlying cancellous and cortical bone ( 3 )

The expansile nature of the lesions cancause pain, swelling, deformity, disruption of growth plates, neurologic symptoms (depending on its location), and pathologic fracture.

Foot are uncommon site of affection. ABC's present about $1 \%$ of all primary bone tumors collectively ( 8 ).

Occurrence in foot is only about $3 \%$ compared to other bones of the body (17). Occurrence within the calcaneum are rare in literature and generally present as chronic heel pain and swelling, but may rarely present as pathologic fracture.

Many osteolytic bone tumor affect calcaneum.So,it is not clinically possible to diagnose osteolytic lesion in calcaneum, making histopathology as the primary investigation and radiology and clinical examination as an accessory protocol for diagnosing $\mathrm{ABC}$.

We should always keep minds the causes osteolytic bone tumor as differential diagnosis-simple bone cyst, chondroblastoma, giant cell tumor (GCT), osteosarcoma, ossifying hematoma or pseudotumor of hemophilia.

\section{Inclusion Criteria:}

As the study is publish only diagnosed 8 cases of anurysmal bone cysts of calcaneum bone as presented to me.
Exclusion criteria:

No other pathology in calcaneum is accepted.

MATERIALSAND METHODS:

In my series $i$ am having 8 cases in last7 years from March 2014 to July 2020.

1.Age variation:

Between 17 years to 29 years.

\section{2. sex variation:}

6 male and 2 female

\section{Side variation:}

5 cases are on left sides and 3 cases are on right sides.

\section{Duration of onset of symptom:}

All cases presented to me between 2 months to 8 months fromthe onset of clinical symptom.

5. socio- economic status:

All 8 cases belongs to lower socioeconomic zone.

\section{Clinical symptom:}

- Chief complaint of swelling in the heel.

- An increase in swelling was associatedwith pain in affected heel. difficulty in walking because of pain.

\section{Clinical signs:}

- Swelling over the medial and lateral aspect of the heel and the skin over the swelling is visibly stretched and shiny.

- Tenderness is present on palpation and local skin temperature is not raised.

- No distal neurovascular deficit is detected.

- No lymphadopathy

\section{Radiological feature:}

Eccentric, expansile, multiloculated lytic lesion of the calcaneum with thin trabeculae traversing the cystic cavity. There was no breach in the cortex.

computed tomography (CT)-

performed in all cases-

A well-defined, expansile lytic lesion with few septations and thinned out cortex noted the on calcaneum

\section{Histological feature-}

Open biopsy were made in all cases to confirm the diagnosis. Reports suggestst cysst grossly consisted of cavities filled with brown altered blood. Histopathological report revealed large blood filled cavities lined by fibrous septa, with occasional osteoclastic giant cells, hemosiderin laden macrophages with a thin rim of bone. 


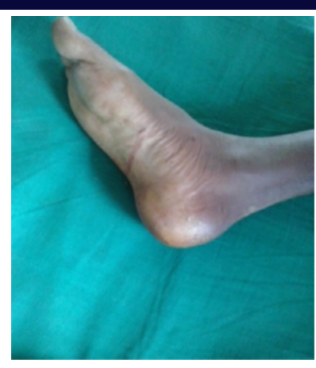

case 1

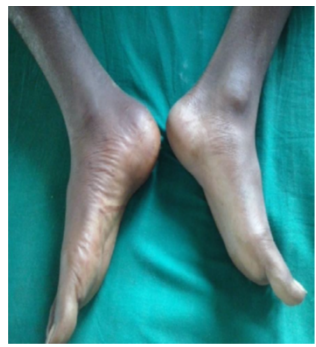

case 2

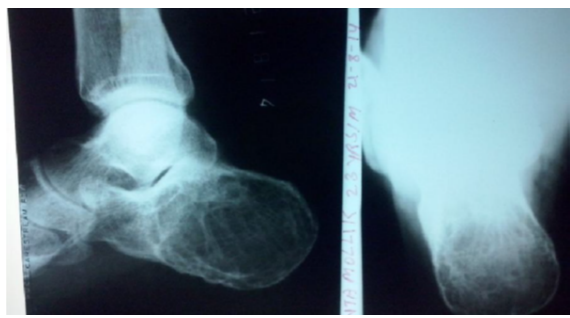

Radiological picture

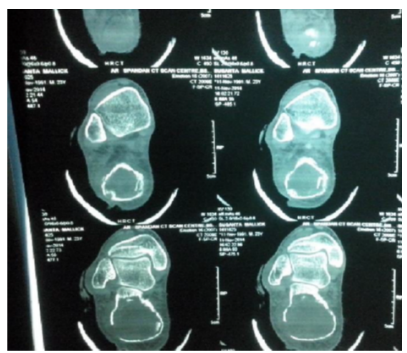

Ct scan picture

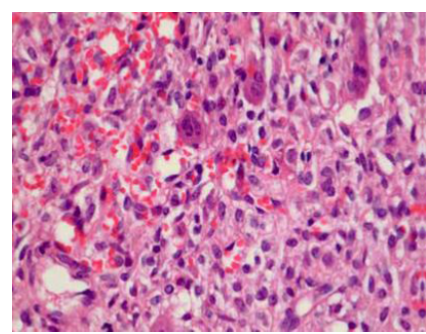

Histological view-

\section{RESULTS OF TREATMENT:}

All patients were treated in same protocol that allows through curettage followed by autogenous cancellous bone grafting and 3 months nonweight bearing rest of the affected limb.Joint mobilization started 7 days after operation to prevent sudek dystrophy.

\section{All patients has healed within 4 months following surgery.}

As the perpose of publication is to report of case series of calcaneum $\mathrm{ABC}$ not the treatment protocol and results, so no further elaboration is done.

\section{DISCUSSION :}

Anurysmal bone cyst is an entity on its having unique clinical, radiological and diagnostic behavior. The exact aetiology of this tumour is unknown. The name is derived from the appearances on macroscope of blood-filled, expansile, sponge-like tumour containing numerous giant cells (13). These lesions can develop on itself or as a result of cystic changes in a pre-existing lesion that causes bone defects (10). A giant cell tumour is the most common cause, occurring in 19\%$39 \%$ of cases and, whatever the aetiology, aneurysmal bone cyst has a close relationship to all the giant cell variants (13)

Many reports suggest that aneurysmal bone cyst is not a true neoplasm since it has been shown to arise in association with other abnormalities of the skeleton (13). Trauma is also considered an initiating factor in the pathogenesis of some cysts in well-documented cases involving acute fracture.

Local hemodynamic alterations to venous obstruction or arteriovenous fistulae that occur after an injury are important in the pathogenesis of an aneurysmal bone cyst .

Aneurysmal bone cysts are seen predominantly in children: $90 \%$ of these lesions occur in patients under 20 years (10)

An aneurysmal bone cyst may occur in patients between the ages of 10 30 years with a peak incidence in those aged 16 years (17). Three quarters of aneurysmal bone cysts occur before epiphyseal fusion has commenced.

$\mathrm{ABC}$ has slight female predominance(13).

The location is usually metaphyseal when the long bones are involved. Purely diaphyseal lesions are seen in $8 \%$ of cases.

Extension into the epiphysis is even more rare (6)

Aneurysmal bone cysts have a predominance for the long bones and lumbar spine (13). Those tumours arising in the spine occur slightly later, between 10 and 20 years. The neural arch is more commonly involved than the body, half of these cases involving more than one vertebra. The prognosis is entirely benign apart from secondary neurological lesions due to spinal canal compression(13).

Regarding the location of the lesions any bone may be affected.

Approximate frequencies by site are listed below:

Skull and mandible (4\%), Spine (16\%),Clavicle and ribs (5\%), Upper extremities (21\%), Pelvis and sacrum (12\%), Femur (13\%); Foot (3\%), Lower leg (23\%) (17)

The most common site involvement in long bones is the metaphyseal region of the knee. Histologically, the lesion consists of multiple blood-filled sinusoid spaces alternating with more solid areas. The solid tissue is composed of fibrous elements containing numerous multinucleated giant cells and is richly vascular. The sinusoids have fibrous walls often containing osteoid tissue or even mature bone (10). The clinical manifestation depends on the specific site of involvement.

Common clinical manifestation includes pain of relatively acute onset pain and swelling that rapidly increases in severity over 6-12 weeks. The local skin temperature may or may not increase, a palpable bony swelling usually be present and movement in the adjacent joint may or may not be restricted; spinal lesions may cause neurologic radiculopathy or quadriplegia and patients may have moderate to severe headaches $(17),(, 6)$.

Diagnostic imaging plays a vital role in patient diagnosis and management. Radiologically, aneurysmal bone cysts are well-defined, cortically based,eccentric rapidly expansile lytic lesions. They can grow large enough to involve the medullary cavity, although their tendency is for eccentric expansion, a so-called blister lesion(12) . A typical area of bone resorption occurs with slight or marked expansion. The size of the lesion can vary between $2 \mathrm{~cm}$ and in gross examples as much as $20 \mathrm{cms}$ in diameter. The overlying cortex is thinned and may be expanded to such a degree that in places it can be identified only by tomography or CT (13).Xray commonly are sufficient for diagnosis. The classically described of an aneurysmal bone cyst in tubular bones includes an eccentric radiolucency and a purely lytic or, occasionally, trabecular process, with its epicenter in the metaphysis of an unfused long bone. The trabeculae in the cyst may create a soap-bubble appearance in the lesion (17). The margins of the lesion are well defined, with a smooth inner margin and a rim of bone sclerosis. The tumor does not usually extend into the epiphyseal plate until after complete fusion, when it may occasionally do so. The expansion or 
ballooning of the cortex occasionally may result in the loss of the sharp definition of its margin and should then be interpreted as an aggressive lesion.

Bone cysts of the calcaneum are rare lesions. These mayinclude a wide spectrum of non-neoplastic cysts, benign or malignant neoplastic lesions ranging from simple bone cyst, $\mathrm{ABC}$ (primary or secondary), chondroblastoma, giant cell tumor (GCT), and an osteosarcoma (especially telangiectatic)(Unni et al., 2010). Clinically, calcaneal cysts are often symptomatic and present with heel pain, although some of these lesions may remain asymptomatic and are detected as incidental findings. Even though there are many typical radiograph, computed tomographic (CT) scan, and magnetic resonance imaging (MRI) findings helps in confirming a diagnosis of $\mathrm{ABC}$, an open biopsy must be performed to confirm thetumors diagnosis(15). When a biopsy is performed, it should be performed by senior surgeon as the sample should ideally include material from the entire lesion; a limited biopsy could easily cause a coexisting lesion to be missed, leaving the patient with a morbid prognosis.

There are various methods of treatment based on the site and size of the lesion, which include curettage, which may be supplemented with various adjuvant therapies such as bone grafting, use of liquid nitrogen, phenol instillation and Poly (methyl methacrylate) (PMMA) cement.

Radiotherapy valuable in spinal lesions where surgery may be considered hazardous. An increasing role, however, has developed for trans-catheter embolisation in the successful management of these tumours. Although relatively rare, there is no reason to assume that $\mathrm{ABCs}$ of the feet will respond to treatment or recur any differently from $\mathrm{ABCs}$ that occur elsewhere in the body. Surgical curettage is sufficient to treat most $\mathrm{ABCs}$ of the feet, including the calcaneum (4).

Series of 8 cases that are found in span 7 years is very rare in description in literature.All patients were young in age with male predominance with left side being the commoner than the right side.All the patients were young in age(second to third decades in life) without having any medical comorbidities.All presented with pain and swelling of the affected heel early in their appearance of symptom.

All patients were investigated radiologically with $\mathrm{x}$-ray and c.t.scan on presentation

All patients were treated with through curettage and autogenous cancellous bone graft taken from iliac crest either from same side or both sides.

All healed nicely in the mean time of 4 months(from 3 months to 5 months).No patient is seen recurrence in the study periods.

\section{CONCLUSION:}

Anurysmal bone cyst of the calcaneum is an very uncommon bone tumor in presentation. Proper diagnosis needs correlating the clinical presentation, anatomical location, radiological picture, and histopathological cofirmation. Allways keeping mind the other causes of lytic lesion not only to exclude other common histological dignosis, but also for choosing the appropriate therapeutic regimen and prognosticates the disease outcome.

\section{REFERENCES}

1. Brastianos P, Gokaslan Z, McCarthy E F. Aneurysmal 2009 bone cysts of the sacrum: a report of ten cases and review of the literature. Iowa Orthop J 29: 74-78.

2. Burch S, Hu S, Berven S. Aneurysmal 2008. bone cysts of the spine. Neurosurg Clin N Am 19(1):41-47

3. Campanacci M, Capanna R, Picci P. Unicameral and aneurysmal1986, bone cysts. Clin Orthop 204: 25-36.

4. Chowdhry M, Chandrasekar CR, Mohammed R, Grimer RJ.2010. Curettage of aneurysmal bone cysts of the feet. FootAnkle Int. 31(2): 131-135.

5. Clayer M. 2008. Injectable form of calcium sulphate astreatment of aneurysmal bone cysts. ANZ J Surg 78(5):366-370.

6. Dahnert, W. 1990. Disease Entities of Bone and Soft Tissue, Radiology Review Manual. Williams and Wilkins;,p 21.

8. Duke Orthopaedics 2007. Wheeless' Textbook of Orthopaedics, Aneurysmal Bone Cyst, Online article, Jan.

9. Enneking W F. Aneurysmal 1983. bone cyst. In: Musculoskeletal tumor surgery. New York: Churchill Livingstone, 1513-29.

10. Greenspan, 1992. A. Orthopedic Radiology. London: Gower Medical Publishing, 14-17

11. Jaffe H L, Lichtenstein L. 1942. Solitary unicameral bone cystwith emphasis on the roentgen picture, the pathologicappearance and the pathogenesis. Arch Surg 44: 10041025

12. Levine, S; Lambiase, R; Pethprapa, C; 2003. Cortical lesions of the Tibia: Characteristics appearances at conventionalradiography. Radiographics, Volume 23, pp 157-177.
13. Sutton, D. 1987. A Textbook of Radiology and Imaging.London: Longman Group 1 , 175-177.

14. Segall L, Cohen-Kerem R, Ngan B Y, Forte V. Aneurysmal bone cysts of the head and neck in pediatric patients: A case series. Int J Pediatr Otorhinolaryngol 21 2008: epub ahead of print

15. Unni KK, Inwards YC. 2010. Conditions that normally simulate primary neoplasms of the bone. In: 16. Unni K K,Inwards Y C, editors. Dahlin's Bone Tumors. 6t edition.Philadelphia: Lippincott Williams and Wilkins;. p. 305-80.

17. Wang EA. Anand MK, Aneurysmal Bone Cyst. 2007 eMedicine,Jan. 